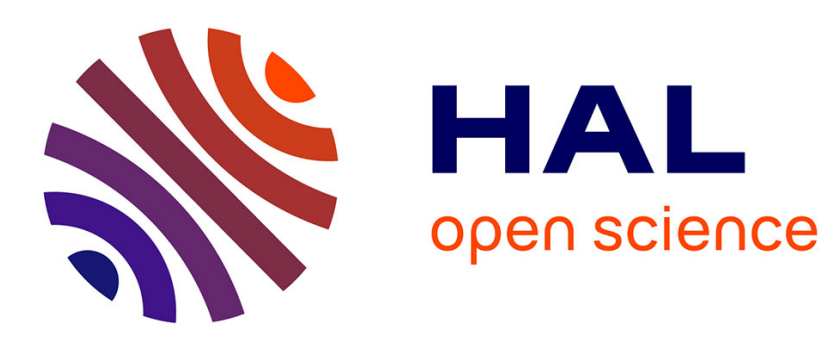

\title{
Prévision de l'énergie digestible des aliments pour le porc: Intérêt du dosage des parois végétales par voie enzymatique
}

\author{
J.M. Perez
}

\section{- To cite this version:}

J.M. Perez. Prévision de l'énergie digestible des aliments pour le porc: Intérêt du dosage des parois végétales par voie enzymatique. Productions Animales, 1990, 3 (3), pp.171-179. hal-00895899

\section{HAL Id: hal-00895899 \\ https://hal.science/hal-00895899}

Submitted on 1 Jan 1990

HAL is a multi-disciplinary open access archive for the deposit and dissemination of scientific research documents, whether they are published or not. The documents may come from teaching and research institutions in France or abroad, or from public or private research centers.
L'archive ouverte pluridisciplinaire HAL, est destinée au dépôt et à la diffusion de documents scientifiques de niveau recherche, publiés ou non, émanant des établissements d'enseignement et de recherche français ou étrangers, des laboratoires publics ou privés. 
INRA Prod. Anim., 1990, 3 (3), $171-179$

\section{J.M. PEREZ}

INRA Laboratoire de Recherches sur l'Elevage du Lapin

BP 27 - 31326 Castanet-Tolosan Cedex
Prévision de l'énergie digestible des aliments pour le porc : Intérêt du dosage des parois végétales par voie enzymatique

La digestibilité de l'énergie conditionne dans une large mesure la valeur énergétique des aliments. Ainsi, près de $90 \%$ des variations de l'« énergie nette porc » selon Noblet et al (1989) sont expliquées par la valeur en énergie digestible. L'efficacité de l'étape digestive dépend directement des teneurs en parois végétales, qui peuvent être estimées désormais par une méthode rapide de dosage mise au point par l'INRA. L'intérêt de cette nouvelle méthode pour la prévision de la valeur énergétique des régimes complexes est discuté comparativement aux autres techniques d'analyse des parois végétales.

Les insuffisances, maintes fois soulignées, du résidu organique de Weende (cellulose brute) ont conduit à rechercher d'autres indicateurs pour caractériser les parois végétales. L'adaptation de la méthode de Van Soest aux aliments concentrés notamment par l'emploi d'amylase

\section{Résumé}

Dans le cadre d'un programme général de recherches sur la prévision de la valeur énergétique des aliments composés destinés au Porc, cette étude avait pour objet d'évaluer l'intérêt d'une nouvelle méthode rapide de dosage des parois végétales par voie enzymatique mise au point par l'INRA (PAR : Parois insolubles dans l'eau).

L'expérimentation portait sur un ensemble de 43 régimes complexes, dont on a cherché en particulier à diversifier la nature des sources de fibres alimentaires. Les mesures de valeur énergétique (ED) ont été réalisées sur des porcs mâles castrés appartenant à la même gamme de poids ( $40 \mathrm{~kg}$ environ), à raison de quatre animaux par régime (172 bilans digestifs au total). Pour accroître la précision des résultats analytiques et la portée des équations de prédiction, les aliments expérimentaux ont fait l'objet de dosages répétés dans plusieurs laboratoires.

Sur la base des critères classiques de sélection des équations (écart-type résiduel, $\mathbf{R}^{2}$ ), les modèles de prédiction mettant en jeu la variable PAR apparaissent dans l'ensemble les plus précis. Cependant, ils ne présentent pas d'avantage décisif par rapport aux modèles de prévision incluant la cellulose brute ou les résidus pariétaux selon Van Soest.

Si on combine les erreurs liées aux modèles (ETR) et les erreurs associées aux analyses (SLAB), les équations avec PAR s'avèrent toujours préférables à celles utilisant les autres indicateurs de parois.

Les meilleurs modèles faisant intervenir PAR aboutissent à des équations de prévision de l'énergie digestible dont l'incertitude totale (équation + analyses) n'excède pas $75 \mathrm{kcal}$ par $\mathrm{kg}$ de matière sèche. L'équation 25 (Lipides, PAR, Cendres, MAT) s'avère la plus intéressante. bactérienne (Giger et al 1979) et ses possibilités d'automatisation (équipement Fibertec) ont permis de développer l'utilisation de cette méthode en alimentation porcine, en particulier pour la prévision de la valeur énergétique des mélanges alimentaires (Perez et al 1984). Néanmoins, cette méthode est loin d'être satisfaisante. Les différentes fractions obtenues par analyse séquentielle (NDF, ADF, AD lignine) ne correspondent pas à des entités biochimiques - bien définies et par voie de conséquence leur signification nutritionnelle est complexe. En outre, la reproductibilité du dosage demeure insuffisante malgré les perfectionnements successifs apportés à la technique d'origine.

Ainsi, dans le cadre d'un programme général sur la prévision de la valeur énergétique des aliments du porc, nous avons évalué sur un nombre conséquent de régimes complexes l'intérêt de la nouvelle méthode de dosage des parois végétales par voie enzymatique mise au point par Carré et Brillouet (1989) sur les aliments destinés aux volailles. Cette étude constitue un des prolongements du travail initial réalisé sur ce thème en concertation avec les industriels de l'alimentation animale et les organismes de développement (Perez et al 1984). Elle a fait l'objet d'une action coordonnée entre l'INRA, l'Institut de Recherches Technologiques AgroAlimentaires des Céréales (IRTAC) et la Direction des Industries Agricoles et Alimentaires (DIAA) du Ministère de l'Agriculture (Carré et al 1988). Nous ne présenterons ici que les principaux résultats en insistant plus particulière- 
ment sur les mérites respectifs des différents critères de parois el sur l'incidence de la variabilité analytique sur la précision des modèles de prévision de la valeur énergétique.

\section{1 / Conditions Expérimentales}

Des précisions sur les méthodes utilisées sont fournies par Perez et al (1984). Les modalités expérimentales peuvent être résumées en quatre points essentiels (voir encadré).

\section{2 / Comparaison des teneurs en parois et en NDF}

Les parois végétales insolubles (PAR) sont censées représenter la totalité des constituants organiques des parois insolubles dans l'eau des cellules végétales: la cellulose, les hémicelluloses et les substances pectiques insolubles dans l'eau, la lignine et les protéines constitutives des parois cellulaires. Comparativement au résidu insoluble enzymatique (méthode PAR), le résidu NDF selon Van Soest sousestime fortement la teneur en parois végétales (de l'ordre de 4 points en moyenne - figure 1). Cette différence provient essentiellement de la solubilisation de la majeure partie des substances pectiques et d'une fraction des hémicelluloses par la solution NDS (détergent neutre) dans la méthode préconisée par Van Soest (Riquet et Tollier 1981). De fait, l'écart le plus important entre les deux méthodes (8 points) s'observe précisément avec un régime renfermant $20 \%$ de pulpes d'agrumes. La relation générale associant, pour les rations étudiées, ces deux critères s'exprime ainsi :

PAR $(\%, M S)=1.09 \mathrm{NDF}(\% \mathrm{MS})+2.99$ $(\mathrm{r}=0.95, \mathrm{ETR}=1.3, \mathrm{n}=43)$.

\section{Les mesures de valeur énergétique (172 bilans digestifs) ont été réalisées sur 43 régimes complexes incluant des sources de parois végétales très diversifiées.}

1 - Cette étude a porté sur un ensemble de 43 régimes complexes formulés à partir d'une série de 20 matières premières. Nous avons cherché en particulier à diversifier les sources de parois végétales : son de blé, pulpes d'agrumes, pellicules de soja, tourteau de pépins de raisins, pulpes de betteraves et coques de tournesol.

2 - Notre objectif de formulation prévoyait des variations de composition suffisamment larges pour ne pas restreindre le champ d'application des équations obtenues, tout en restant dans des limites proches des conditions pratiques (tableau 1). En outre, nous avons fait en sorte de rendre aussi indépendantes que possible les différentes caractéristiques analytiques des régimes de manière à faciliter ultérieurement l'interprétation des régressions multiples.

3 - Les mesures de digestibilité ont été réalisées en cages individuelles suivant la méthode habituelle utilisée à l'INRA. Au total, 172 bilans digestifs ont été effectués sur des porcs mâles castrés de $40 \mathrm{~kg}$ de poids vif environ, à raison de quatre animaux par régime.

4 - Pour accroître la précision des résultats analytiques et la portée des équations de prédiction de la valeur énergétique, les aliments expérimentaux ont été analysés dans plusieurs laboratoires. Dix laboratoires au total ont participé à la chaîne d'analyse. Ainsi, les dosages ont été répétés 7 fois pour les cendres et la cellulose brute, 6 fois pour l'azote, 4 fois pour l'amidon (Ewers) et les lipides (sans hydrolyse) et 2 fois pour les sucres totaux. Les constituants pariétaux selon Van Soest ont été déterminés dans deux laboratoires après harmonisation des procédures (Giger et al 1979, 1987). Les parois végétales insolubles dans l'eau (PAR) ont été dosées à l'INRA (ToulouseAuzeville) selon la méthode décrite par Carré et Brillouet (1989) et normalisée par lAFNOR (1989). Son principe consiste en une solubilisation de l'amidon par empesage et dégradation par une alpha-amylase thermostable (Termamyl) ; les protéines sont ensuite solubilisées par une solution de détergent et dégradées à l'aide d'une protéase (pronase) ; puis, les lipides sont solubilisés par le détergent et un mélange chloroforme-méthanol.

Tableau 1. Teneurs moyennes et plages de variation des principales caractéristiques des régimes

\begin{tabular}{|c|c|c|c|}
\hline Caractéristiques (1) & Moyenne & Minimum & Maximum \\
\hline Cendres & 6,8 & 5,4 & 8,5 \\
\hline Matières azotées totales (MAT) & 18,6 & 13,9 & 21,8 \\
\hline Lipides (LIP1) (2) & 3,6 & 1,2 & 9,8 \\
\hline Amidon Ewers & 47,8 & 33,8 & 61,8 \\
\hline Sucres totaux & 5,3 & 2,8 & 16,6 \\
\hline Cellulose brute (CB) & 4,8 & 2,3 & 11,1 \\
\hline NDF & 12,7 & 7,4 & 20,9 \\
\hline $\mathrm{ADF}$ & 5,5 & 2,7 & 13,0 \\
\hline Lignine (ADL) & 1,0 & 0,1 & 3,5 \\
\hline PAR (3) & 16,8 & 10,4 & 27,1 \\
\hline Energie brute (EB) & 4358 & 4110 & 4689 \\
\hline Energie digestible (ED) & 3580 & 3252 & 4058 \\
\hline
\end{tabular}

(1) Exprimées en \% (ou en $\mathrm{kcal} / \mathrm{kg}$ ) par rapport à la matière sèche

(2) LIP1 : lipides extraits par l'other diéthylique sans hydrolyse acide préalable.

(3) PAR : parois végétales insolubles dans l'eau. 
Figure 1. Comparaison des teneurs en parois insolubles (PAR) et en NDF des régimes.

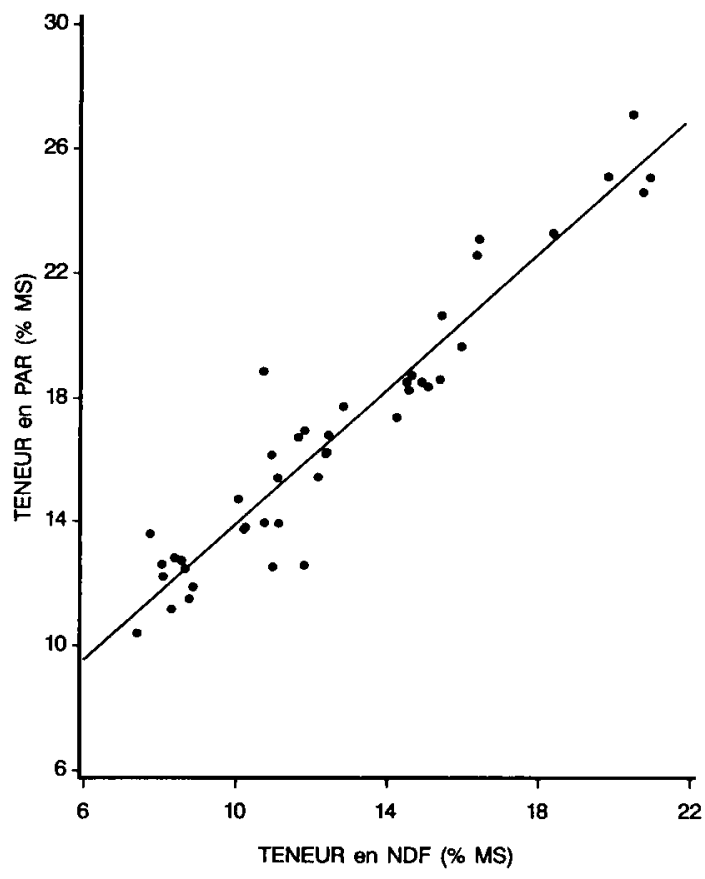

\section{3 / Valeur prédictrice des critères de parois}

Les régressions linéaires simples du CUD apparent de l'énergie (CUDE) des régimes sur les différents indicateurs de parois sont rapportées dans le tableau 2. On constate que les variables PAR, NDF, ADF et cellulose brute fournissent, dans l'ensemble, des équations dont la précision est très voisine. L'équation faisant intervenir PAR présente le coefficient de variation résiduel $(\mathrm{CVr})$ le plus faible $(2 \%)$. Ce critère explique à lui seul $84 \%$ des variations du CUDE, alors qu'à l'opposé, la lignine n'explique que la moitié de la variance.
Le pouvoir prédicteur d'un critère ne se limite pas à la valeur du coefficient de corrélation et/ou de l'écart-type résiduel. Ainsi, l'analyse détaillée (non rapportée ici) des écarts aux régressions simples précédentes conduit à privilégier parmi les indicateurs de «fibres » ceux qui prennent en compte plus complètement les parois végétales (PAR ou NDF). En effet, les critères cellulose brute (Weende) et ADF (Van Soest) entraînent des biais importants dans la prédiction du CUDE (et de la valeur énergétique) des régimes complexes pour des valeurs élevées (surestimation de l'ED) ou faibles (sousestimation de l'ED) du rapport parois totales/ fraction lignocellulose : cas des aliments riches en son de blé (régime 7) ou en coques de soja (régime 5) par exemple.

Pour l'ensemble des 43 régimes, l'effet dépressif de la cellulose brute (CB) sur la digestibilité de l'énergie correspond à un taux de diminution moyen de 1,7 point $(s b=0,13)$ par point supplémentaire de CB dans la matière sèche. Ce taux se révèle intermédiaire entre les valeurs extrêmes rapportées antérieurement (Henry et Perez 1983) avec les aliments simples: 3 points environ pour les sources riches en hémicelluloses (issues de meunerie) et 1 point pour les matières premières à forte proportion de lignocellulose dans les parois (graines et sous-produits de légumineuses). On retrouve précisément ces valeurs dans notre étude avec les régimes renfermant des proportions croissantes de son de blé (18 et $36 \%$ ) ou de coques de soja (12 et $24 \%$ ). Cette absence de relation générale étroite entre le CUD de l'énergie et la teneur en cellulose brute explique les discordances entre les auteurs dans l'estimation de la pente de la droite de régression $\mathrm{CUDE}=\mathrm{a}-\mathrm{b}$ CB pour les régimes complexes (Drennan et Maguire 1970, Schiemann et al 1971, King et Taverner 1975, Schneider et Kirchgessner 1977, Just 1982, Noblet et al 1989). Dans ce cas en effet, la valeur (b) et la précision (sb) du coefficient de régression sont très dépendantes de la nature et de la diversité des matières premières composant les régimes expérimentaux. Ces résultats traduisent le fait
L'analyse des écarts aux équations de régression conduit à privilégier parmi les indicateurs de fibres ceux qui prennent en compte plus complètement les parois végétales (PAR ou NDF).

Tableau 2. Prévision du CUD apparent de l'énergie à partir d'un seul indicateur de parois : régressions simples (1)

\begin{tabular}{|c|c|c|c|c|c|c|}
\hline Critères de parois & Constante & $\begin{array}{r}\text { Coeff } \\
\text { de rég }\end{array}$ & $\begin{array}{l}\text { cient } \\
\text { ession }\end{array}$ & $\begin{array}{l}\mathbf{R}^{2} \\
(3)\end{array}$ & $\begin{array}{c}\text { ETR } \\
\text { (3) }\end{array}$ & $\begin{array}{c}\text { CVr } \\
\text { (3) }\end{array}$ \\
\hline Cellulose brute & 90,50 & $-1,72$ & $(0,13)$ & 0,816 & 1,84 & 2,2 \\
\hline $\begin{array}{l}\text { NDF } \\
\text { Lignocellulose (ADF) } \\
\text { Hémicelluloses (NDF-ADF) } \\
\text { Cellulose (ADF-ADL) } \\
\text { Lignine (ADL) }\end{array}$ & $\begin{array}{l}95,28 \\
90,57 \\
92,70 \\
89,69 \\
86,16\end{array}$ & $\begin{array}{l}-1,03 \\
-1,52 \\
-1,47 \\
-1,66 \\
-4,01\end{array}$ & $\begin{array}{l}(0,07) \\
(0,12) \\
(0,27) \\
(0,19) \\
(0,59)\end{array}$ & $\begin{array}{l}0,833 \\
0,799 \\
0,412 \\
0,652 \\
0,529\end{array}$ & $\begin{array}{l}1,75 \\
1,92 \\
3,29 \\
2,53 \\
2,94\end{array}$ & $\begin{array}{l}2,1 \\
2,3 \\
4,0 \\
3,1 \\
3,6\end{array}$ \\
\hline Parois ( PAR) & 97,47 & $-0,91$ & $(0,06)$ & 0,844 & 1,70 & 2,0 \\
\hline
\end{tabular}

(1) $Y=a+b X$

Y: CUD apparent de l'énergie (en \%)

$\mathrm{X}$ : teneur exprimée en \% de la matière sèche

(2) Entre parenthèses : écart-type (Sb) du coefficient de régression (b)

(3) $\mathrm{R}^{2}$ : coefficient de détermination ; ETR : écart-type résiduel ;

CVr : coefficient de variation résiduel (\%) 
que la cellulose brute est, comme la lignocellulose au détergent acide (ADF), un descripteur incomplet et non représentatif des parois cellulaires végétales. Ils indiquent donc les limites importantes de ce type de critère pour la prévision de la valeur énergétique des mélanges alimentaires.

A l'inverse, l'inconvénient des critères globaux PAR et NDF réside dans leur inaptitude à refléter l'hétérogénéité de composition pariétale des régimes complexes. A cet égard, il faut souligner les différences spécifiques entre les volailles et les porcs. Pour ces derniers en effet, il faut tenir compte non seulement du taux de parois, mais aussi du degré variable de dégradation des différentes fractions pariétales. Cela explique le gain de précision faible pour la prévision de la valeur énergétique (et du CUDE) de

Figure 2. Influence des teneurs en parois (PAR) sur la digestibilité de l'énergie des régimes $(n=43)$.

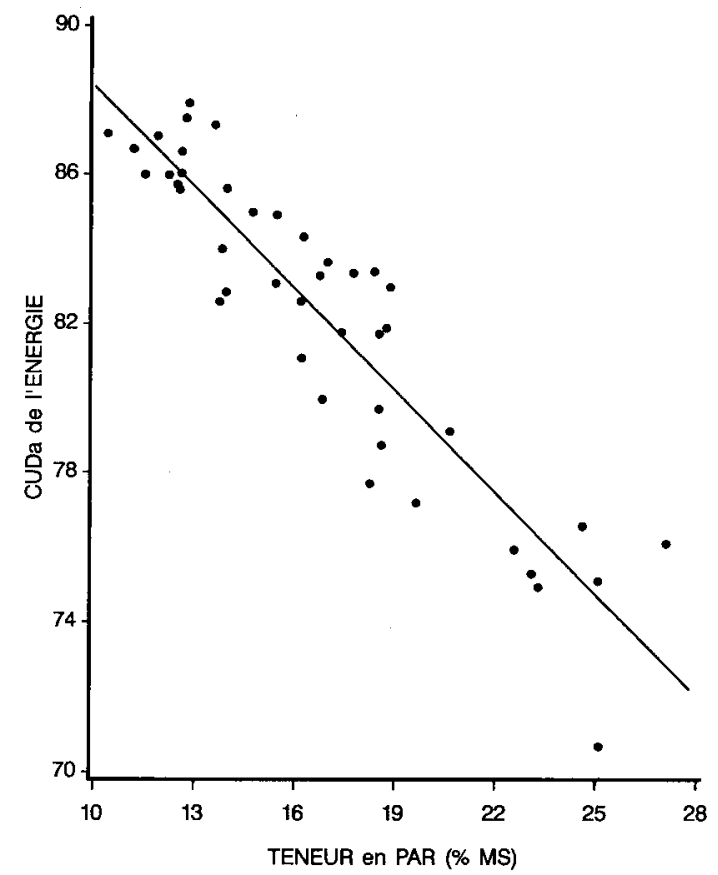

PAR comparativement au NDF. Dans le même sens, si on constate logiquement que l'équation reliant le CUDE au NDF surestime les régimes riches en substances pectiques (par exemple, le régime 8 renfermant $20 \%$ de pulpes d'agrumes\} car ces composés ne sont pas pris en compte dans le résidu NDF de Van Soest, on observe en revanche que la relation générale CUDE $=f(P A R)$ sous- estime l'utilisation digestive de l'énergie de ces mêmes régimes, en raison d'une plus forte digestibilité du « résidu insoluble enzymatique » comparativement aux autres régimes moins pourvus en substances pectiques très digestibles chez le Porc!

La relation linéaire décroissante (figure 2) entre le CUDE et la teneur en parois (PAR), indique un taux de diminution moyen de 0,9 point $(\mathrm{sb}=0,06)$ par point supplémentaire de parois dans la matière sèche. Le taux correspondant est de 1 point $(\mathrm{sb}=0,07)$ pour le résidu NDF en conformité avec les résultats bibliographiques $(0,8$ à 1,2 point selon King et Taverner 1975, Henry 1977, Noblet et al 1989). Il convient de noter la plus grande homogénéité (et la meilleure précision intra-essai) des estimations de ces coefficients de régression comparativement aux valeurs rapportées pour la cellulose brute. Cela reflète le rôle primordial des teneurs en parois totales comme facteur explicatif des variations de la digestibilité de l'énergie.

En fait, le taux de diminution du CUDE varie aussi, mais dans une moindre mesure seulement, en fonction de la composition des parois elles-mêmes en raison des différences d'utilisation digestive des diverses fractions pariétales, comme nous l'avons évoqué précédemment. Ainsi, d'après nos résultats (tableau 3), la combinaison des différentes fractions pariétales améliore la précision des modèles de prédiction de la digestibilité de l'énergie des mélanges alimentaires, ce qui corrobore d'autres observations (Nehring et Uhlemann 1972, Schneider et Kirchgessner 1977). L'association des variables " lignocellulose " (ADF) et "hémicelluloses"

Tableau 3. Prévision du CUD apparent de l'énergie à partir d'une combinaison de critères de parois (1).

\begin{tabular}{|c|c|c|c|c|c|c|c|c|c|}
\hline $\begin{array}{r}\text { Critères } \\
\text { Constante }\end{array}$ & NDF & $\begin{array}{l}\text { Ligno- } \\
\text { cellulose } \\
\text { (ADF) }\end{array}$ & $\begin{array}{c}\text { Cellulose } \\
\text { (ADF - ADL) }\end{array}$ & $\underset{\text { (ADL) }}{\text { Lignine }}$ & $\begin{array}{c}\text { Hémi- } \\
\text { celluloses } \\
\text { (NDF - ADF) }\end{array}$ & $\begin{array}{l}\text { Parois } \\
\text { (PAR) }\end{array}$ & $\begin{array}{l}\mathbf{R}^{2} \\
(3)\end{array}$ & $\begin{array}{c}\text { ETR } \\
(3)\end{array}$ & $\begin{array}{l}\text { CVr } \\
\text { (3) }\end{array}$ \\
\hline 93,95 & & $\begin{array}{c}-1,29(2) \\
(0,11)\end{array}$ & & & $\begin{array}{l}-0,65 \\
(0,15)\end{array}$ & & 0,861 & 1,62 & 2,0 \\
\hline 93,64 & & & $\begin{array}{l}-1,05 \\
(0,13)\end{array}$ & $\begin{array}{r}-2,28 \\
(0,33)\end{array}$ & $\begin{array}{r}-0,62 \\
(0,14)\end{array}$ & & 0,889 & 1,47 & 1,8 \\
\hline 94,38 & $\begin{array}{r}-0,85 \\
(0,08)\end{array}$ & & & $\begin{array}{l}-1,47 \\
(0,39)\end{array}$ & & & 0,878 & 1,52 & 1,9 \\
\hline 96,24 & & & & $\begin{array}{l}-1,62 \\
(0,34)\end{array}$ & & $\begin{array}{c}-0,74 \\
(0,06)\end{array}$ & 0,901 & 1,37 & 1,7 \\
\hline
\end{tabular}

(1) $Y=a+b_{1} X_{1}+b_{2} X_{2}+\ldots$

$Y$ : CUD apparent de l'énergie (en \%)

$\mathrm{X}$ : teneurs exprimées en $\%$ de la matière sèche.

(2) Coefficient de régression (b). Entre parenthèses : écart-type (Sb) du coefficient.

(3) $R^{2}$ : coefficient de détermination ; ETR : écart-type résiduel ; CVr : coefficient de variation résiduel (\%). 
Tableau 4. Les modèles de prévision de la valeur énergétique (ED) avec 2 et 3 facteurs (1)

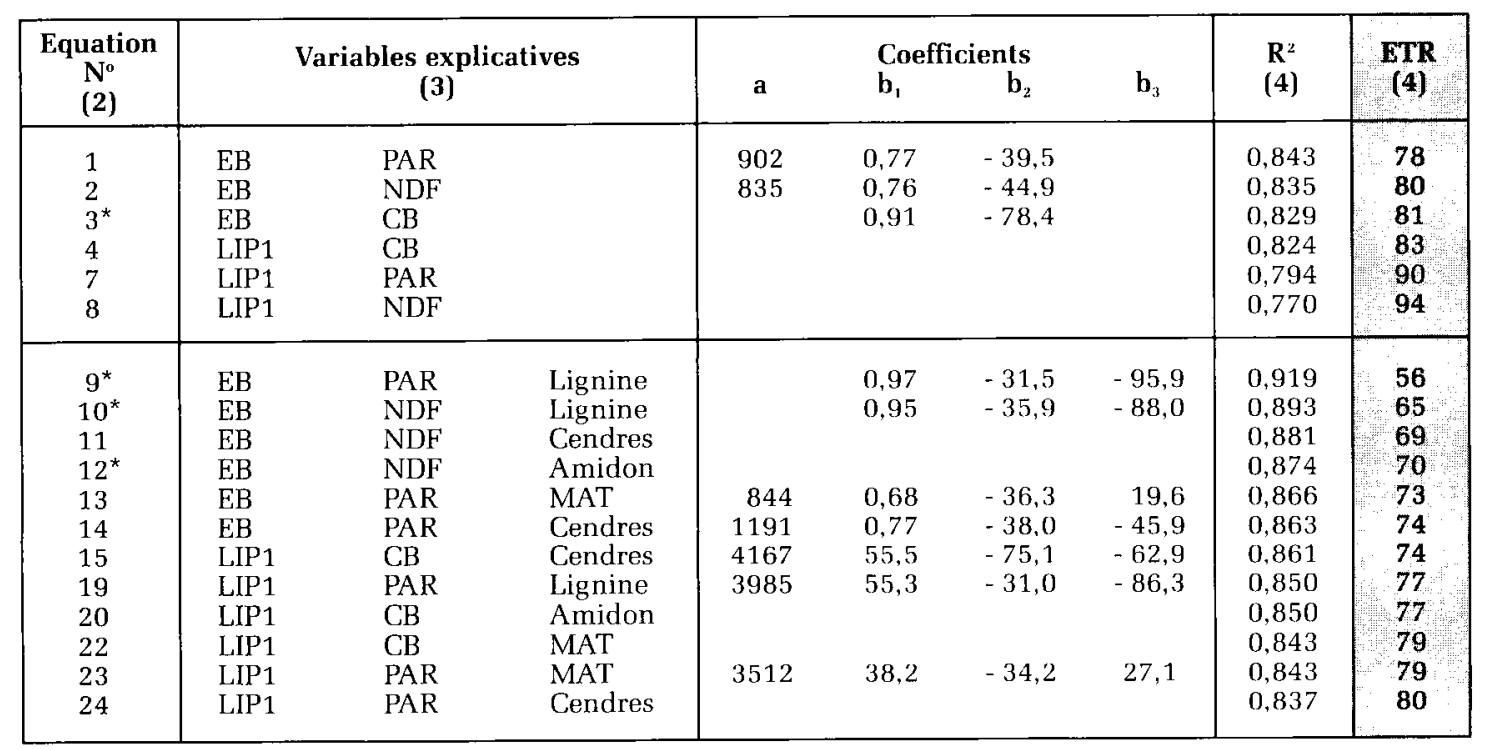

(1) $\mathrm{Y}=\mathrm{a}+\mathrm{b}_{1} \mathrm{X}_{1}+\mathrm{b}_{2} \mathrm{X}_{2}+\ldots$; $\mathrm{Y}$ : Energie digestible en kcal/kg MS ; $\mathrm{X}$ : Teneurs exprimées en \% (ou en kcal) par rapport à la matière sèche.

$(2)^{\star}$ : équations avec ordonnée à l'origine non significativement différente de zéro : valeurs de $\mathrm{R}^{2}$ et FT'R recalculées en annulant la constante.

(3) voir tableau $1 ;(4)$ voir tableau 2

de Van Soest (NDF-ADF) explique dans notre étude $86 \%$ des variations du CUDE. En séparant la fraction lignocellulose en ces deux composantes, on améliore encore significativement la précision ( $89 \%$ de la variance expliquée par rapport à $83 \%$ avec le NDF seul).

De la même façon, en combinant le critère PAR avec un indicateur d'indigestibilité des parois, en l'occurrence la lignine au détergent acide (ADL), on aboutit à une équation très précise (CV résiduel $<1,7 \%$ ) qui explique $90 \%$ des variations de digestibilité de l'énergie. Cet amalgame entre la méthode PAR et le schéma de Van Soest peut surprendre. Mais, on pourrait très bien envisager d'affiner le dosage enzymatique des parois, pour son application spécifique en alimentation porcine, en le complétant par une dernière étape incluant un traitement approprié du résidu final par l'acide sulfurique concentré. Les avantages a priori d'une telle démarche au plan nutritionnel ne doivent pas faire perdre de vue les risques analytiques d'une telle procédure séquentielle plus complexe avec les conséquences éventuelles sur la fiabilité et la reproductibilité de la méthode (cf. Van Soest!). Une autre possibilité réside dans la détermination directe des teneurs en lignines en complément de l'estimation des parois totales (PAR). La signification et les limites des méthodes de dosage du résidu ligneux ont été discutées en détail par ailleurs (Giger 1985).

\section{4 / Les modèles de prévision de la valeur énergétique}

D'une façon générale parmi les indicateurs de parois, le critère PAR s'avère toujours le plus performant dans les modèles de prévision de la valeur en énergie digestible (ED) des régimes. Le résidu NDF est également préférable à la cellulose brute.

Dans le tableau 4, nous présentons les équations à 2 facteurs qui se sont révélées les plus intéressantes. Elles associent l'énergie brute, ou les lipides, avec un indicateur de parois. L'équation 1 la plus précise $(\mathrm{ETR}=78 \mathrm{kcal} / \mathrm{kg}$ MS) combine l'énergie brute (EB) et PAR. Ces deux variables expliquent ensemble $84 \%$ de la variation de l'énergie digestible des régimes. L'analyse des résidus de la régression montre que cette équation a toutefois l'inconvénient de surestimer la valeur énergétique des régimes riches en lignine. Dans le même tableau, sont rassemblées également les meilleures équations à 3 facteurs. Nous avons écarté les équations présentant un écart-type résiduel (ETR) supé-

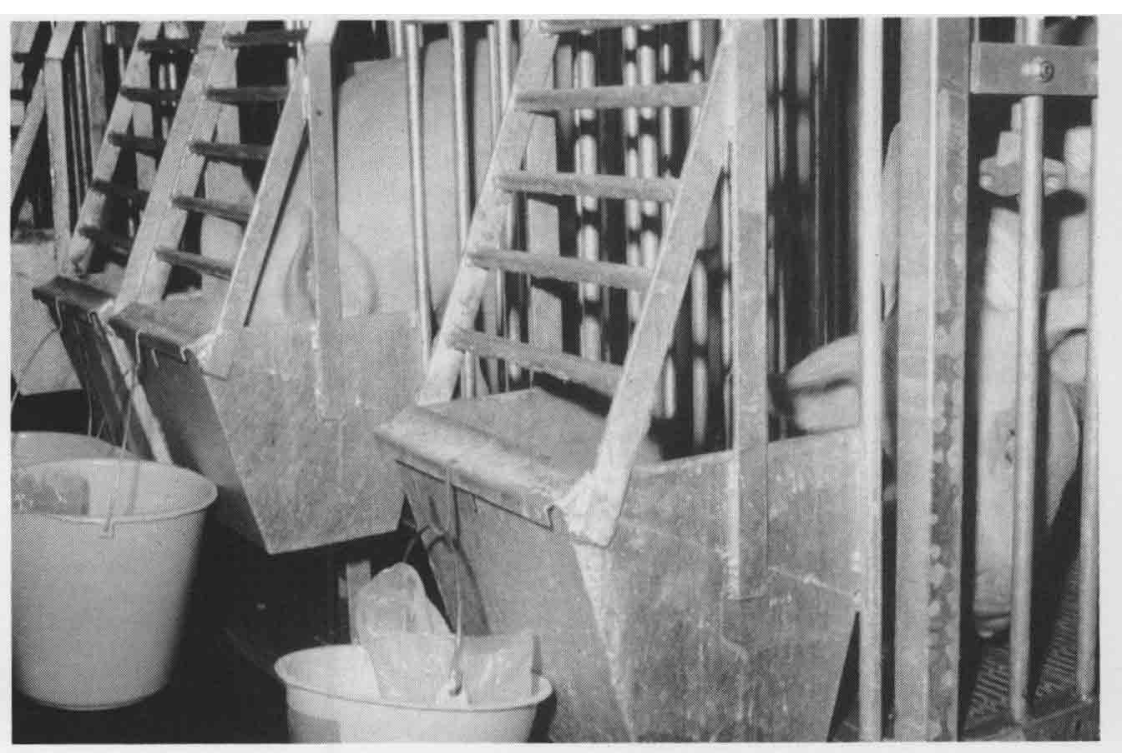

Détermination in vivo de la valeur énergétique : collecte totale des excreta (fèces et urine) pendant 10 jours consécutifs sur des porcs mâles castrés placés dans des cages spécialement équipée pour les bilans digestifs (4 animaux par régimel. 
Tableau 5. Les modèles de prévision de la valeur énergétique (ED) avec 4 et 5 facteurs.

\begin{tabular}{|c|c|c|c|c|c|c|c|c|c|c|c|c|c|}
\hline $\begin{array}{c}\text { Equation } \\
N^{0} \\
(1)\end{array}$ & \multicolumn{5}{|c|}{ Variables explicatives } & \multicolumn{6}{|c|}{ Coefficients } & $\mathbf{R}^{2}$ & RTA \\
\hline 25 & LIP1 & PAR & Cendres & MAT & & 3899 & 39,2 & $-30,2$ & $-93,1$ & 36,4 & & 0,919 & 58 \\
\hline 26 & LIP1 & MAT & Amidon & Sucres & & 390 & 76,9 & 51,9 & 36,3 & 40,5 & & 0,916 & 58 \\
\hline 27 & LIP1 & PAR & MAT & Amidon & & 2460 & 49,3 & $-25,0$ & 40,1 & 12,9 & & 0,911 & 60 \\
\hline $28 *$ & $\mathrm{~EB}$ & NDF & Lignine & Cendres & & & & & & & & 0,911 & 60 \\
\hline 30 & $\mathrm{~EB}$ & PAR & Cendres & MAT & & 1245 & 0,65 & $-32,8$ & $-67,6$ & 27,8 & & 0,906 & 62 \\
\hline 32 * & $\mathrm{EB}$ & NDF & ADF & Lignine & & & & & & & & 0,905 & 62 \\
\hline 33 & LIP1 & $\mathrm{CB}$ & Cendres & MAT & & & & & & & & 0,905 & 62 \\
\hline 34 & LIP1 & $\mathrm{CB}$ & MAT & Amidon & & 2416 & 57,0 & $-52,7$ & 33,3 & 12,4 & & 0,904 & 62 \\
\hline 35 * & $\mathrm{EB}$ & PAR & MAT & Amidon & & & & & & & & 0,901 & 63 \\
\hline 36 & $\mathrm{~EB}$ & $\mathrm{NDF}$ & Cendres & MAT & & & & & & & & 0,897 & 65 \\
\hline 38 & EB & $\mathrm{NDF}$ & Lignine & Amidon & Sucres & & & & & & & 0,939 & 60 \\
\hline $39 *$ & EB & PAR & Lignine & Cendres & MAT & & & & & & & 0,935 & 52 \\
\hline 40 & LIP1 & PAR & Cendres & MAT & Amidon & 3160 & 45,3 & $-26,1$ & $-63,2$ & 41,0 & 7,5 & 0,935 & 52 \\
\hline 41 & LIP1 & PAR & Lignine & Cendres & MAT & 3879 & 46,3 & $-27,3$ & $-47,3$ & $-81,3$ & 31,7 & 0,934 & 53 \\
\hline 42 & LIP1 & $\mathrm{CB}$ & MAT & Amidon & Sucres & & & & & & & 0,933 & 53 \\
\hline 43 & LIP1 & PAR & MAT & Amidon & Sucres & & & & & & & 0,933 & 53 \\
\hline 47 & LIP1 & PAR & Lignine & MAT & Amidon & & & & & & & 0,927 & 56 \\
\hline
\end{tabular}

(1) Voir tableau 4.

Sur la base des critères classiques de sélection des équations, les différents indicateurs de parois conduisent à des modèles de prédiction de précision assez comparable. rieur à $80 \mathrm{kcal} / \mathrm{kg}$ MS et pour alléger la présentation, seuls les coefficients de régression des principales équations sont précisés. A partir du modèle à 2 facteurs associant EB (ou les lipides) et un indicateur de parois, les variables supplémentaires les plus intéressantes sont la lignine et les cendres, et dans une moindre mesure l'amidon et les protéines brutes. L'équation 9 (EB, PAR, Lignine) est particulièrement précise (ETR $=56 \mathrm{kcal} / \mathrm{kg} \mathrm{MS}$ ) et avec trois facteurs seulement elle explique $92 \%$ des variations de la valeur énergétique.

Le tableau 5 fournit les équations de régression à 4 variables explicatives. Nous n'avons retenu que les équations dans lesquelles les variables avaient une contribution significative par rapport aux modèles précédents à 3 facteurs, et dont les écarts-types résiduels étaient inférieurs à $65 \mathrm{kcal} / \mathrm{kg} \mathrm{MS}$. Aucune équation à 4 facteurs ne s'avère plus précise que l'équation $\mathrm{n}^{\circ} 9$ (EB, PAR, Lignine). Les équations les plus performantes avec PAR $(25,27,30,35)$ incluent EB (ou les lipides), les cendres (ou l'amidon) et les protéines brutes. Les équations 26 (Lipides, MAT, Amidon, Sucres) et 33 (Lipides, CB, Cendres, MAT) sont les meilleures équations faisant intervenir les critères classiques d'analyse. Nous avons essayé d'améliorer encore la prédiction en utilisant des modèles à 5 facteurs. Dans ces conditions, les variables supplémentaires présentent le plus souvent des coefficients non significatifs.

\section{5 / Les meilleures équations de prédiction}

Le classement précédent (tableaux 4 et 5 ) est basé uniquement sur les erreurs liées au modèle (ETR, $\mathrm{R}^{2}$ ). Il est sensiblement modifié si l'on tient compte également de l'incidence des erreurs analytiques sur la précision des équations.
L'influence de la variabilité analytique sur la précision des modèles de prévision de la valeur énergétique a été discutée par Fisher (1982) et Alderman (1985). Pour juger de l'intérêt d'un modèle de prédiction, il est nécessaire d'estimer son « incertitude totale » (STOT) qui est la résultante de l'incertitude de l'ajustement des données expérimentales (variation résiduelle du modèle proposé ou ETR) et de l'incertitude liée aux erreurs analytiques (SLAB). Cette dernière se calcule à partir des valeurs de reproductibilité (écarts-types inter-laboratoires) des dosages (tableau 6). Les valeurs de reproductibilité $\left(\mathrm{s}_{\mathrm{i}}\right)$ adoptées pour les critères classiques d'analyse tiennent compte des données des chaînes BIPEA (Sauvant et Schmidt 1984), complétées par les résultats des différents circuits analytiques réalisés sur les aliments destinés aux porcs et aux volailles (Fisher 1983, Perez et al 1984, Cooke 1987, Morgan et al 1987, Carré et al 1988, Noblet et al 1989). Pour le critère PAR, nous avons fixé à $6 \%$ la valeur du coefficient de variation (CV) inter-laboratoires, ce qui correspond à la valeur maximum enregistrée par Carré et Brillouet (1989), le CV moyen obtenu dans cette étude (11 aliments analysés dans 7 laboratoires] étant de $4 \%$.

Dans ces conditions, le classement des équations (tableau 6) à partir du cumul des incertitudes liées au modèle et aux analyses conduit à privilégier les modèles incluant la variable PAR : sur les quinze meilleures équations, dix font intervenir ce critère. Parmi l'ensemble des équations à 5 facteurs, seules trois équations $(40,41,47)$ s'avèrent un peu plus précises que les équations les plus performantes à 4 facteurs. Elles ont l'inconvénient de faire appel à 5 variables explicatives et le faible gain de précision obtenu ne justifie pas le coût analytique supplémentaire qu'elles entraînent. Les équations 25 (Lipides, PAR, Cendres, MAT) et 30 (EB, PAR, Cendres, MAT) sont particulièrement intéressantes, car elles mettent en jeu simultanément les deux meilleurs « indicateurs éner- 
gétiques », EB (ou les lipides) et PAR, avec deux autres paramètres (MAT et cendres) dont la détermination est de toute façon indispensable pour l'appréciation générale de la qualité de l'aliment.

Comme le montrent des données complémentaires sur la digestibilité des constituants pariétaux (non rapportées ici) et l'analyse des résidus des régressions, ce type d'équation utilisant PAR comme seule caractéristique des parois aura cependant tendance à surestimer la valeur énergétique des régimes à fortes teneurs en parois indigestibles (notamment riches en lignine) et à sous-estimer celle des aliments renfermant des proportions importantes d'hémicelluloses. Les écarts entre les valeurs ED prédites et les valeurs mesurées sont illustrés sur la figure 3 pour l'équation 25.

Par ailleurs, il est intéressant de remarquer sur le tableau 6, que certaines équations parmi les meilleures dans le classement précédent se trouvent dévaluées dès lors qu'elles comportent des caractéristiques analytiques dont la reproductibilité est mauvaise. Il en est ainsi de l'équation 9 incluant la lignine et de l'équation 26 incluant les sucres. Enfin, il convient de nuancer l'intérêt des équations faisant intervenir le critère PAR, dans la mesure où la reproductibilité de ce nouveau type d'analyse est encore difficile à cerner. Un calcul de simulation faisant varier le CV inter de ce critère montre néanmoins que l'équation 25 (Lipides, PAR, Cendres, MAT) demeure encore intéressante par rapport à l'équation la plus performante $\left(\mathrm{n}^{\circ}\right.$ 33) utilisant les critères classiques d'analyse (Lipides, CB, Cendres, MAT), si le CV interlaboratoires de PAR ne dépasse pas $8 \%$.
Figure 3. Energie digestible des régimes: Ecarts entre valeurs mesurées et valeurs calculées à l'aide de l'équation 25 (Lipides, PAR, Cendres, MAT).

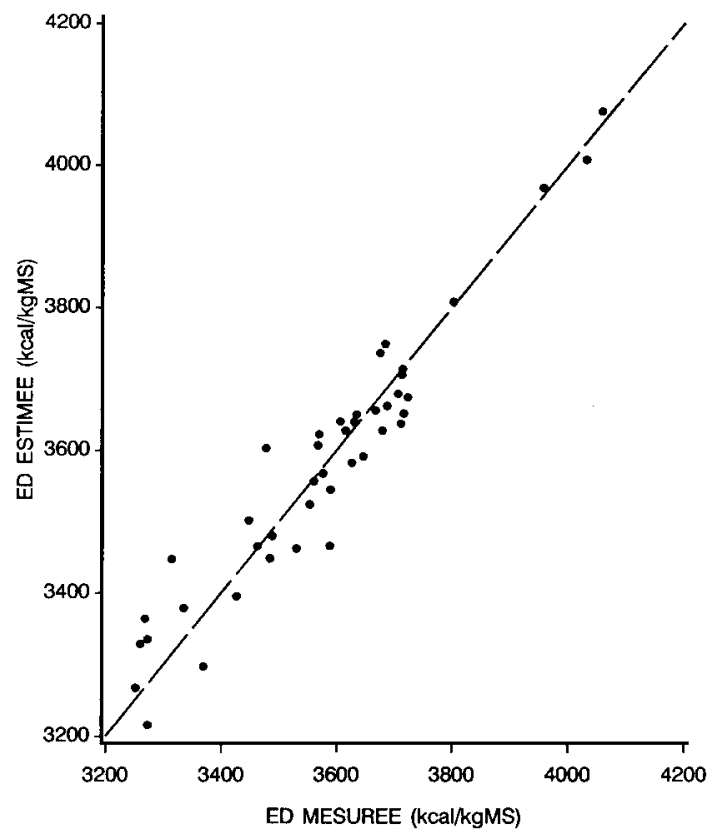

\section{Conclusions}

1 - Sur la base des critères classiques de sélection des équations (écart-type résiduel, $\mathrm{R}^{2}$ ), les modèles de prédiction de la valeur énergétique mettant en jeu la variable PAR (Parois insolubles dans l'eau) apparaissent dans l'ensemble

Tableau 6. Les meilleures équations: prise en compte du cumul des incertitudes liées aux modèles et aux analyses.

\begin{tabular}{|c|c|c|c|c|c|c|c|c|c|}
\hline \multirow{4}{*}{$\begin{array}{c}\begin{array}{c}\text { Equation } \\
\mathbf{N}^{\mathbf{0}}\end{array} \\
40 \\
41 \\
47\end{array}$} & \multirow{4}{*}{$\begin{array}{c}\text { Nombre } \\
\text { facteurs }\end{array}$} & \multicolumn{5}{|c|}{ Variables explicatives } & \multirow{4}{*}{$\begin{array}{c}\begin{array}{c}\text { ETR } \\
\text { (1) }\end{array} \\
52 \\
53 \\
55\end{array}$} & \multirow{4}{*}{$\begin{array}{c}\text { SLAB } \\
\text { (2) } \\
40 \\
42 \\
38\end{array}$} & \multirow{4}{*}{$\frac{\frac{510 T}{(8)}}{\frac{66}{67}}$} \\
\hline & & LIP1 & PAR & Cendres & MAT & Amidon & & & \\
\hline & & LIP1 & PAR & Lignine & Cendres & MAT & & & \\
\hline & & LIP1 & PAR & Lignine & MAT & Amidon & & & \\
\hline 25 & 4 & LIP1 & PAR & Cendres & MAT & 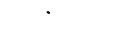 & 58 & 43 & 72 \\
\hline 27 & 4 & LIP1 & PAR & MAT & Amidon & & 60 & 39 & 72 \\
\hline 34 & 4 & LIP1 & $\mathrm{CB}$ & MAT & Amidon & & 62 & 40 & 74 \\
\hline 33 & 4 & LIP1 & $\mathrm{CB}$ & Cendres & MAT & & 62 & 44 & 87 \\
\hline 30 & 4 & EB & PAR & Cendres & MAT & & 62 & 49 & 79 \\
\hline 35 & 4 & $\mathrm{~EB}$ & PAR & MAT & Amidon & & 63 & 48 & 79 \\
\hline 26 & 4 & LIP1 & MAT & Amidon & Sucres & & 59 & 68 & 90 \\
\hline 32 & 4 & $\mathrm{~EB}$ & NDF & $\mathrm{ADF}$ & Lignine & & 62 & 67 & 91 \\
\hline 9 & 3 & $\mathrm{~EB}$ & PAR & Lignine & & & 56 & 60 & 82 \\
\hline 15 & 3 & LIP1 & CB & Cendres & & & 74 & 46 & 87 \\
\hline 23 & 3 & LIP1 & PAR & MAT & & & 79 & 38 & 8 \\
\hline 19 & 3 & LIP1 & PAR & Lignine & & & 77 & 43 & 88 \\
\hline 20 & 3 & LIP1 & $\mathrm{CB}$ & Amidon & & & 77 & 43 & 80 \\
\hline 13 & 3 & $\mathrm{~EB}$ & PAR & MAT & & & 73 & 51 & 69 \\
\hline 22 & 3 & LIP1 & CB & MAT & & & 79 & 42 & 00 \\
\hline 14 & 3 & $\mathrm{~EB}$ & PAR & Cendres & & & 74 & 52 & 90 \\
\hline 24 & 3 & LIP1 & PAR & Cendres & & & 80 & 42 & 91 \\
\hline
\end{tabular}

(1) (2) (3) exprimés en $\mathrm{kcal} / \mathrm{kg}$ MS

(1) ETR : écart-type résiduel

(2) SLAB : écart-type associé aux erreurs analytiques

SLAB : $\left[\sum b_{i}^{2} s_{i}^{2}\right]^{1 / 2}$, avec $s_{i}$ écarts-types inter-laboratoires ;

Valeurs de s retenues (en \% ou kcal par rapport à la matière sèche) : Cendres (0.3), MAT (0.5), Lipides (0.3), Amidon (1.4), Sucres (0.8), CB (0.5), NDF (1.8), ADF (0.7), Lignine (0.3), PAR (1.0), EB (44).

(3) STOT : écart-type total cumulant les erreurs liées au modèle (ETR) et aux analyses (SLAB).

Si on combine les erreurs liées aux modèles et les erreurs liées aux analyses, les équations avec la variable PAR (Parois insolubles dans l'eau) s'avèrent toujours préférables à celles mettant en jeu les autres méthodes de dosage des constituants pariétaux. 
les plus précis. Cependant, ils ne présentent pas d'avantage décisif par rapport aux modèles de prévision incluant la cellulose brute ou les résidus pariétaux selon Van Soest.

2 - Si on combine les erreurs liées aux modèles (ETR) et les erreurs associées aux analyses (SLAB), les équations avec PAR s'avèrent toujours préférables à celles utilisant les autres indicateurs de parois.

3 - Les meilleurs modèles faisant intervenir PAR aboutissent à des équations de prévision de l'énergie digestible dont l'incertitude totale (équation + analyses) n'excède pas $75 \mathrm{kcal}$ par $\mathrm{kg}$ de matière sèche. L'équation 25 (Lipides, PAR, Cendres, MAT) s'avère la plus intéressante.

4 - Le choix des meilleurs prédicteurs étant conditionné dans une large mesure par la précision analytique, des études de très grande ampleur sont encore nécessaires pour mieux préciser la reproductibilité de la procédure enzymatique PAR comparativement aux autres techniques d'analyse des parois végétales, et confirmer ainsi l'intérêt de cette nouvelle méthode rapide de dosage des fibres pour la prévision de la valeur énergétique des aliments destinés au Porc.

\section{Remerciements}

Celte étude a fait l'objet d'une convention de recherches entre l'INRA, la Direction des Industries Agricoles et Alimentaires (DIAA) et l'Institut de Recherches Agro-alimentaires des Céréales (IRTAC) Elle a bénéficié du soutien financier du Ministère de l'Agriculture (DGAL).

Nous adressons nos remerciements à Muriel Ségura (INRA- Toulouse) pour le dosage des parois végétales par voie enzymatique, ainsi qu'aux différents laboratoires ayant participé aux analyses chimiques des aliments expérimentaux dans la première phase de ce programme (INRA-St Gilles, INA-PG, Sanders, Guyomarc'h, ITCF, CCPA, UCAAB, UFAC et le Servico de la répression des fraudes de Rennes)

\section{Références bibliographiques}

AFNOR, 1989. Abments des animax. Détemination de la teneur en parois végétales insolubles dans l'eau. Norme expérimentale NF V 18 111. AFNOR, Paris.

ALDERMAN G., 1985. Prediction of the energy value of compound feeds. In : Haresign W., Cole D.J.A. (ed.), Recent advances in animal nutrition, 3-52. Butterworths, London.

CARRE B., BRILLOUET J.M., 1989. Determination of water-insoluble cell walls in feeds : interlaboratory study J. Assoc. Off. Anal. Chem., 72, 463-467.

CARRE B. PEREZ J M. LEBAS F, 1988. Mesure des fibres végétales dans les aliments pour animaux. Compte rendu final, Convention de recherches DIAA/IRTAC $n^{\prime}$ 86/02. Institut de Recherches Technologiques AgroAlimentaires des Céréales, Paris.
COOKE B.C., 1987. The impact of declaration of the metabolizable energy $(\mathrm{ME})$ value of poultry feeds. In : Haresign W., Cole D.J.A. (ed.), Recent advances in animal nutrition, 19 - 26. Butterworths, London.

DRENNAN P., MAGUIRE M.F., 1970. Prediction of the digestible and metabolisable energy content of pig diets from their fibre content. Ir. J. Agric. Res., 9, 197-202.

FISHER C., 1982. Energy values of poultry compound feeds. Occasional publication $n^{\circ} 2$. ARC Poultry Research Centre, Roslin, $109 \mathrm{p}$.

FISHER C., 1983. Report on a ring-test to evaluate the proposed EEC equation for the declaration of $\mathrm{ME}$ values, poultry. Occasional publication n" $n^{\prime \prime}$. ARC Poultry Research Centre, Roslin, $34 \mathrm{p}$.

GIGER S., 1985. Revue sur les méthodes de dosage de la lignine utilisées en alimentation animale. Ann. Zootech. 34, 85-122.

GIGER S., SAUVANT D., DORLEANS M., MORANDFEHR P., 1979. Détermination semi-automatique des constituants membranaires des aliments concentrés par la méthode de Van Soest. 30th Annual Meeting of the EAAP, Harrogate, England, July 1979. Commission on Animal Feeding, N3.5.

GIGER S., THIVEND P., SAUVANT D., DORLEANS M, JOURNAIX P., 1987. Etude de l'influence préalable de différentes enzymes amylolytiques sur la teneur en résidu NDF d'aliments du bétail. Ann. Zootech., 36, 39-48.

HENRY Y., 1977. Prediction of energy values of feeds for swine from fiber content. In : Fonnesbeck P.V., Harris L.E., Kearl L.C. (ed.), Proc. 1st Int. Symp. Feed Composition, Animal Nutrient Requirements and Computerization of Diets, Logan, 11-16 July 1976, 270-281. Utah State Univ.

HENRY Y., PEREZ J.M., 1983. Les systèmes d'évaluation de l'énergie dans l'alimentation du porc (2). Les Dossiers de l'Elevage, 5 (2), 49-64.

JUST A., 1982. The net energy value of balanced diets for growing pigs. Livest. Prod. Sci., 8, 541-555.

KING R.H., TAVERNER M.R., 1975. Prediction of the digestible energy in pig diets from analyses of fibre contents. Anim. Prod., 21, 275-284.

MORGAN C.A., WHITTEMORE C.T., PHILLIPS P. CROOKS P., 1987. The prediction of energy value of compounded pig foods from chemical analysis. Anim. Feed Sci. Technol., 17, 81-107.

NEHRING K., UHLEMANN H., 1972. Uber den Futterwert der Getreidemüllereiprodukte. 4. Die Beziehungen zwischen Gehalt an Gerüstsubstanzen und Verdaulichkeit. Arch. Tierernähr., 22, 59-84.

NOBLET I., FORTUNE H., DUBOIS S., HENRY Y., 1989

Nouvelles bases d'estimation des teneurs en énergie digestible, métabolisable et nette des aliments pour le porc. INRA, Paris, $106 \mathrm{p}$

PEREZ J.M., RAMIHONE R., HENRY Y., 1984. Prédiction de la valeur énergétique des aliments composés destinés au porc : étude expérimentale. INRA, Paris, $95 \mathrm{p}$.

RIQUET A.M., TOLIIER M.T., 1981. Détermination des teneurs en polyosides pariétaux et en lignine des cellules végétales selon différentes méthodes. II. Signification et limites d'utilisation de ces méthodes. Lebensm.-Wiss. Technol., 14, 113-117.

SAUVANT D., SCHMIDT M., 1984. Variations des résultats analytiques et prévision de la valeur énergétique des concentrés et sous-produits pour le ruminant. In : La prévision de la valeur énergétique des aliments concentrés et des sous-produits assimilés, CAAA INA-PG, 13 déc. 1984, H1-H25. ADEPRINA ed., Paris.

SCHNEIDER R., KIRCHGESSNER M., 1977. Zur Schätzung der Verdaulichkeit der organischen Substanz von Schweinefuttermitteln mittels der Gehalte verschiedener Zellwandfraktionen. Z. Tierphysiol. Tierernähr. Futtermittelk., 39, 211-219.

SCHIEMANN R., NEHRING K., HOFFMANN L., JENTSCH W., CHUDY A., 1971. Energetische Futterbewertung und Energienormen. V.E.B. Deutscher Landwirtschaftsverlag, Berlin, $344 \mathrm{p}$. 


\section{Summary}

Prediction of the energy value of pig feeds by enzymatic cell wall analysis.

The purpose of this study performed in connection with the general research programme on prediction of the energy value of compound feeds for pigs was to estimate the advantage of a new rapid enzymatic method for cell wall analysis developed by INRA (WICW : waterinsoluble cell wall).

The experiment involved 43 complex diets containing various types of dietary fibres. The feed energy value (DE) was measured in castrated male pigs of the same weight range (about $40 \mathrm{~kg}$ ) using four animals per diet (a total of 172 digestive balances). With the aim of increasing the accuracy of the analytical data and the impact of prediction equations, the experimental feeds underwent several analyses in various laboratories.

Main results :

1 - On the basis of classical criteria for choice of equations for prediction of feed energy value (residual standard deviation, $\mathbf{R}^{2}$ ), the models involving the WICW variable seemed to be the most accurate. However, they did not show a very marked advantage relative to prediction models involving crude fibre or cell wall residues according to Van Soest.

2 - When combining the errors related to the models (RSD) and the errors associated with the analyses (SLAB), equations with WICW always proved to be better than those using the other cell wall indicators.

3 - With the best models using WICW, the total uncertainty of equations for prediction of digestible energy (equation + analyses) did not exceed $75 \mathrm{kcal}$ per kg dry matter. Equation 25 (Lipids, WICW, Ashes, Crude Protein) proved to be the most interesting.

4 - As the choice of the best predictors depends to a large extent on the accuracy of analysis, further large-scale investigations are required for better determining the reproductibility of the WICW method as compared to other techniques for plant cell wall analysis and confirm its interest for prediction of the energy value of pig feeds.

PEREZ J.M., 1990. Prévision de l'énergie digestible des aliments pour le porc: Intérêt du dosage des parois végétales par voie enzymatique. INRA Prod. Anim., 3 (3), 171 - 179. 